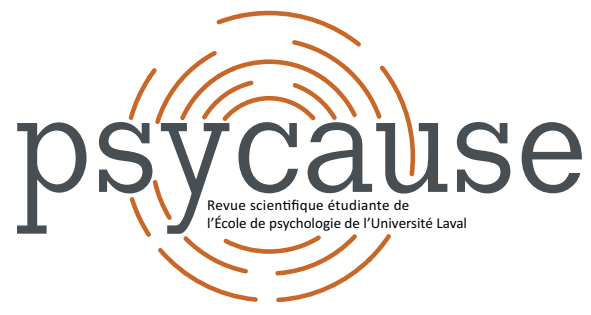

\title{
PSYCAUSE
}

Revue scientifique étudiante de l'École de psychologie de l'Université Laval

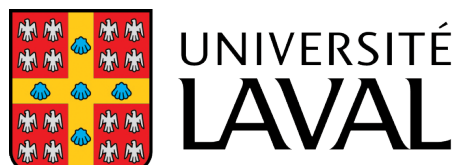

Faculté des sciences sociales École de psychologie

\section{VALIDATION FRANCOPHONE DES QUESTIONNAIRES DE SOMMEIL AUPRÈS DES ÉTUDIANTS-ATHLÈTES DU QUÉBEC}

\author{
Amandine GAGNON-HÉBERT ${ }^{1, *}$, Mikael VERRAULT ${ }^{1}$, Adèle JOBIN-THÉBERGE ${ }^{1}$ \\ Jonathan CHAREST ${ }^{1}$ et Célyne H. BASTIEN ${ }^{1}$ \\ ${ }^{1}$ École de psychologie, Université Laval \\ *amandine.gagnon-hebert.1@ulaval.ca
}

\section{Pour citer l'article}

Gagnon-Hébert, A., Verrault, M., Jobin-Théberge, A., Charest, J., \& Bastien, C. H. (2019). Validation francophone des questionnaires de sommeil auprès des étudiants-athlètes du Québec. Psycause: Revue scientifique étudiante de l'École de psychologie de l'Université Laval, 9(2), 25-26. 


\section{VALIDATION FRANCOPHONE DES QUESTIONNAIRES DE SOMMEIL AUPRÈS DES ÉTUDIANTS-ATHLÈTES DU QUÉBEC}

Amandine GAGNON-HÉBERT ${ }^{1, \star}$, Mikael VERRAULTT ${ }^{1}$, Adèle JOBIN-THÉBERGE ${ }^{1}$, Jonathan CHAREST ${ }^{1}$ et Célyne H. BASTIEN ${ }^{1}$

${ }^{1}$ École de psychologie, Université Laval

* amandine.gagnon-hebert.1@ulaval.ca

\section{Mots-clés: Sommeil des athlètes; Validation transculturelle; Qualité du sommeil; Hygiène du sommeil}

Les étudiants-athlètes courent un risque accru de développer des difficultés de sommeil comparativement à la population étudiante générale (Simpson, Gibbs \& Matheson, 2017). Ils auraient en moyenne entre 6,5 et 6,7 heures de sommeil quotidiennement. Cette quantité de sommeil est nettement inférieure aux neuf à dix heures de sommeil recommandées pour les sportifs d'élite (Mah, Mah, Kezirian \& Dement, 2011). Un manque de sommeil chez les étudiants-athlètes peut avoir des conséquences importantes sur la santé psychologique et physique des athlètes (Fullagar et coll., 2015). Bien que plusieurs études se soient intéressées au sommeil de cette population et à ses conséquences, aucune d'entre elles n'avaient à sa disposition des questionnaires pour l'évaluation du sommeil validés auprès des athlètes. Les questionnaires d'évaluation du sommeil auprès de la population générale ne sont effectivement pas adaptés aux athlètes puisqu'ils ne prennent pas en compte le mode de vie spécifique des athlètes caractérisé par leur impératif à conjuguer leurs obligations académiques, sociales et sportives (Samuels, James, Lawson \& Meeuwisse, 2016). L'Athlete Sleep Screening Questionnaire (ASSQ; Samuels et coll., 2016) et l'Athlete Sleep Behavior Questionnaire (ASBQ; Driller et coll., 2018) sont deux questionnaires qui ont récemment été développés pour répondre à ce besoin d'outils validés considérant la haute demande physiologique et psychologique quotidienne des athlètes.
Cependant, ces derniers ne sont disponibles qu'en anglais, ce qui restreint leur utilisation à la population anglophone. L'objectif de cette étude est donc de valider la version française de l'ASSO et de l'ASBO.

\section{Méthode}

À deux reprises, dans un intervalle de sept jours, 203 étudiants-athlètes (132 hommes et 71 femmes) dont la moyenne d'âge est de 20,5 $\pm 2,1$ ans ont complété l'ASSO, I'ASBO et I'Index de sévérité de l'insomnie (ISI). Les caractéristiques de l'échantillon se trouvent dans le Tableau 1. L'ASSQ et l'ASBO sont les deux questionnaires étant l'objet de la validation. Ils visent respectivement l'évaluation de la qualité et de l'hygiène du sommeil chez les athlètes. Quant à I'ISI, il s'agit d'un questionnaire visant à évaluer la nature, la sévérité et l'impact subjectifs des symptômes associés à I'insomnie (Bastien, Vallières \& Morin, 2001). Un agenda du sommeil a également été complété par les participants afin de recueillir divers renseignements sur le patron et les habitudes de sommeil des étudiants-athlètes (voir Figure 1).

Plusieurs analyses des propriétés psychométriques ont été effectuées sur le Score de difficulté de sommeil (SDS) de I'ASSO. II s'agit d'un sous-ensemble des items de ce questionnaire. Les mêmes analyses ont également été effectuées considérant tous les items de l'ASBO à chacun des temps

Tableau 1

Nombre d'athlètes par sport, sexe, niveau de scolarité et moment de la saison sportive.

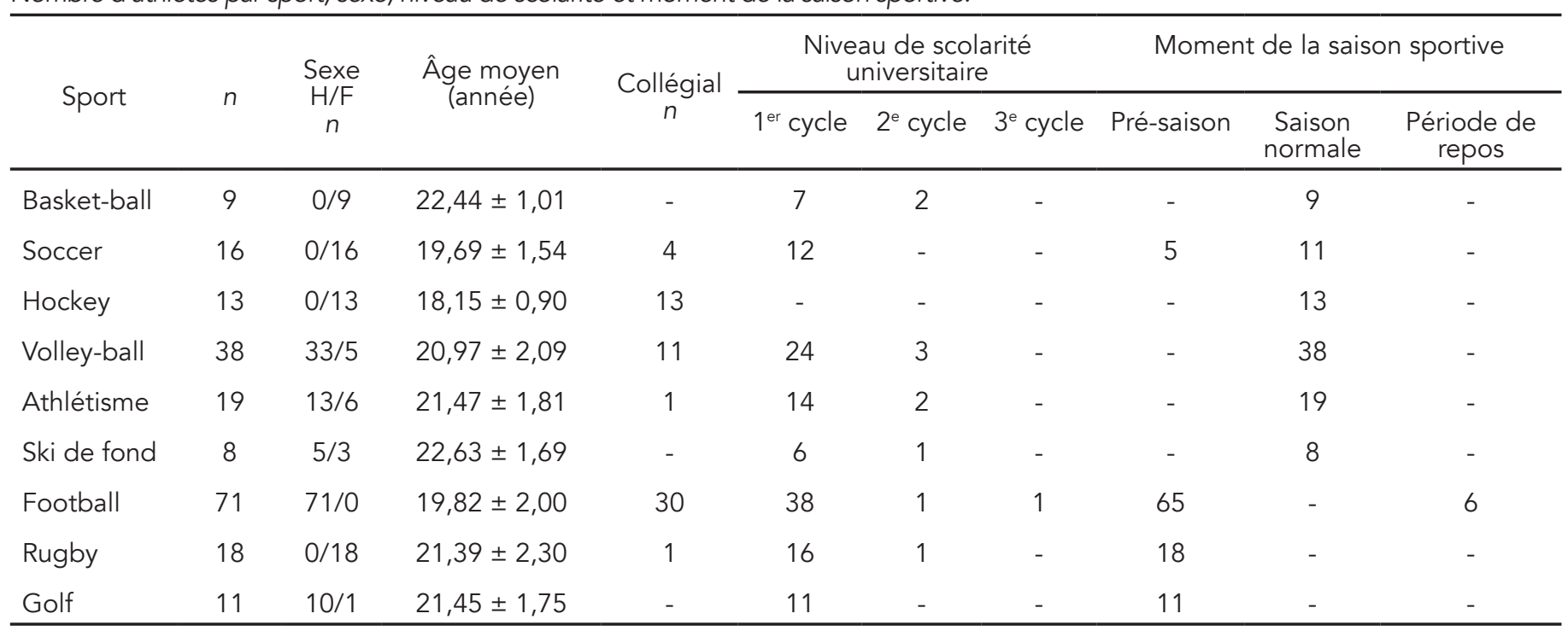




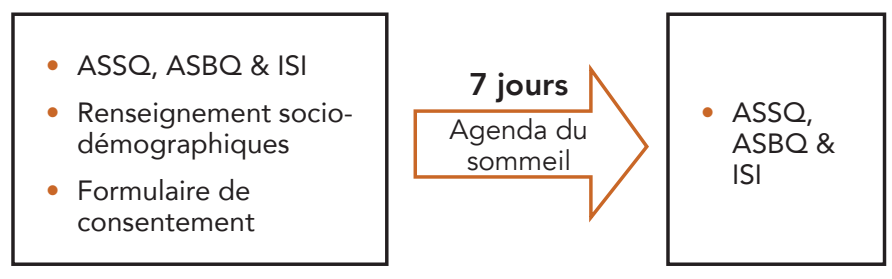

Figure 1. Passation de l'Athlete Sleep Screening Questionnaire (ASSQ), de l'Athlete Sleep Behavior Questionnaire (ASBQ) et de l'Index de sévérité de I'insomnie (ISI) à deux reprises dans un intervalle de 7 jours.

de mesures et ce, en utilisant l'ISI et l'agenda du sommeil comme mesures comparatives.

\section{Résultats et discussion}

\section{ASSQ}

Le SDS identifie correctement les athlètes qui ne possèdent pas de difficultés de sommeil. II possède une meilleure spécificité (88\%-92\%) qu'une meilleure sensibilité (67\%-85\%), et ce, aux deux temps de mesure. En ce qui a trait à la cohérence interne du questionnaire, elle se révèle insuffisante pour une utilisation clinique telle que déterminée par l'alpha de Cronbach ( $\alpha=0,56-0,62)$. De plus, certaines corrélations item-total corrigées du SDS sont inférieures à $r=0,30$, ce qui est sous le seuil recommandé par Ferketich (1991). Ces derniers résultats suggèrent que les items composant le SDS se composent d'items évaluant différents construits. La validité externe est également insuffisante puisque les items du SDS obtiennent des corrélations de force faible à moyenne avec les items de l'agenda du sommeil $(r=-0,36-0,16)$. Le SDS de l'ASSO pourrait toutefois refléter un ensemble de difficultés liées à l'insomnie en raison des corrélations de force moyenne entre le SDS et le score global de I'ISI ( $r=$ $0,58-0,62)$. Enfin, le SDS se caractérise par une stabilité temporelle adéquate $(r=0,73)$.

\section{ASBQ}

La cohérence interne de la version française de l'ASBO est acceptable et supérieure à celle de la version originale telle que mesurée par l'alpha de Cronbach ( $\alpha=0,68-0,72$; Driller et coll., 2018). Toutefois, la majorité des corrélations item-total corrigées sont insuffisantes selon les critères de Ferketich (1991). L'ASBO obtient également une stabilité temporelle adéquate $(r=0,72)$.
La cohérence interne des deux questionnaires et la validité convergente du SDS de I'ASSO s'expliqueraient, entre autres, par des lacunes de la traduction française ainsi qu'à la structure trop contraignante de l'énoncé de certains items ne permettant pas une évaluation optimale du construit. Cependant, les versions françaises de l'ASSO et de l'ASBO possèdent des propriétés psychométriques suffisantes pour une utilisation non clinique, de recherche ou de dépistage auprès des athlètes présentant des difficultés de sommeil. La force de ces deux outils réside principalement dans la forte stabilité temporelle de ces derniers. Une retraduction de certains items est suggérée afin d'améliorer les propriétés psychométriques des deux questionnaires.

\section{Références}

Bastien, C. H., Vallières, A., \& Morin, C. M. (2001). Validation of the Insomnia Severity Index as an outcome measure for insomnia research. Sleep Medicine, 2(4), 297-307. doi:10.1016/S1389-9457(00)00065-4

Driller, M. W., Mah, C. D., \& Halson, S. L. (2018). Development of the athlete sleep behavior questionnaire: a tool for identifying maladaptive sleep practices in elite athletes. Sleep Science, 11(1), 37. doi:10.5935/1984-0063.20180009

Ferketich, S. (1991). Focus on psychometrics: Aspects of item analysis. Research in Nursing \& Health, 14, 165-168. doi:10.1002/nur.4770140211

Fullagar, H. H., Skorski, S., Duffield, R., Hammes, D., Coutts, A. J., \& Meyer, T. (2015). Sleep and athletic performance: The effects of sleep loss on exercise performance, and physiological and cognitive responses to exercise. Sports Medicine, 45(2), 161-186. doi:10.1007/s40279-014-0260-0

Mah, C. D., Mah, K. E., Kezirian, E. J., \& Dement, W. C. (2011). The effects of sleep extension on the athletic performance of collegiate basketball players. Sleep, 34(7), 943-950. doi:10.5665/SLEEP.1132

Samuels, C., James, L., Lawson, D., \& Meeuwisse, W. (2016). The Athlete Sleep Screening Questionnaire: A new tool for assessing and managing sleep in elite athletes. British Journal of Sports Medicine, 50(7), 418-422. doi:10.1136/ bjsports-2014-094332

Simpson, N. S., Gibbs, E. L., \& Matheson, G. O. (2017). Optimizing sleep to maximize performance: implications and recommendations for elite athletes. Scandinavian Journal of Medicine \& Science in Sports, 27(3), 266-274. doi:10.1111/sms.12703

\section{Pour citer l'article}

Gagnon-Hébert, A., Verrault, M., Jobin-Théberge, A., Charest, J., \& Bastien, C. H. (2019). Validation francophone des questionnaires de sommeil auprès des étudiants-athlètes du Québec. Psycause: Revue scientifique étudiante de l'École de psychologie de I'Université Laval, 9(2), 25-26. 